\title{
Materiales compuestos de superficie sólida fabricados a partir de un jarabe de polimetilmetacrilato, trihidróxido de aluminio y cargas minerales naturales
}

\author{
Solid surface composite materials manufactured \\ from syrup of polymethyl methacrylate, alumina \\ trihydrate and natural mineral fillers
}

Adan Arribasplata Seguin ${ }^{1}$, Kelly Lucas Lizano ${ }^{1}$, Juan Rueda Sánchez ${ }^{2,3}$, Julio Acosta Sullcahuamán ${ }^{1}$

\footnotetext{
${ }^{1}$ Sección Ingeniería Mecánica, Pontificia Universidad Católica del Perú, Av. Universitaria 1801,San Miguel, Lima, Perú. ${ }^{2}$ Sección Física, Pontificia Universidad Católica del Perú, Av. Universitaria 1801,San Miguel, Lima, Perú.

${ }^{3}$ Dirección de Gestión de la Investigación - DGI, Pontificia Universidad Católica del Perú, Av. Universitaria 1801,San Miguel, Lima, Perú.

e-mail: adan.arribasplata@pucp.pe,klucas1@pucp.edu.pe,jrueda@pucp.edu.pe,jacosta@pucp.edu.pe
}

\section{RESUMEN}

Se desarrolló un material compuesto a partir de un jarabe de polimetilmetacrilato (PMMA), trihidróxido de aluminio (ATH) y cargas minerales naturales $(\mathrm{CMN})$ con propiedades similares al material comercial Krion ${ }^{\circledR}$. Con este propósito, se fabricaron materiales compuestos en dos etapas: En la primera etapa, se realizó la polimerización parcial, en masa, vía radicales libres del metacrilato de metilo (MMA) para obtener un jarabe de polimetilmetacrilato y se determinaron los parámetros de la polimerización necesarios para evitar el efecto de autoaceleración y, al mismo tiempo, lograr un jarabe de un porcentaje de conversión de PMMA capaz de evitar la sedimentación de partículas de ATH. En la segunda etapa, se fabricaron y caracterizaron diversas muestras de materiales compuestos a partir de la mezcla del jarabe de PMMA obtenido anteriormente, partículas de ATH y CMN, con el propósito de determinar los parámetros adecuados para lograr propiedades similares al Krion ${ }^{\circledR}$. Se ha demostrado que, para evitar la autoaceleración y la sedimentación de ATH, se debe lograr un jarabe con un porcentaje de conversión de MMA en PMMA entre $20 \%$ y $25 \%$ en peso y la polimerización parcial debe realizarse con $0,1 \%$ en peso de peróxido de benzoilo (PBO) y $0,2 \%$ en peso de etilenglicol dimercaptoacetato (EGDM) a $85^{\circ} \mathrm{C}$ durante 90 minutos. Similarmente, para obtener propiedades similares al Krion ${ }^{\circledR}$, los parámetros para la fabricación de un material compuesto de PMMA reforzado con partículas de ATH y CMN son: 40\% jarabe / 60\% ATH / \%CMN variable según el tipo de carga, $0,1 \%$ en peso de PBO adicional, $2,0 \%$ en peso de etilenglicol dimetacrilato (EGDMA) y 13 horas de curado en un horno a $85^{\circ} \mathrm{C}$. Las propiedades del material compuesto con solo ATH fabricado bajo estas condiciones son: $53 \mathrm{MPa}$ de resistencia a flexión, $8054 \mathrm{MPa}$ de módulo elástico en flexión y 91 HRM de dureza. La calidad estética mejora con la incorporación de CMN pero las propiedades mecánicas disminuyen.

Palabras clave: superficie sólida, polimerización radicalar, polimetilmetacrilato, trihidróxido de aluminio, carga mineral natural.

\section{ABSTRACT}

A composite material, which properties are similar to the commercial material Krion $\AA$, was manufactured using polymethyl methacrylate (PMMA) syrup, alumina trihydrate (ATH) and natural mineral fillers (CMN). For this purpose, composites have been manufactured in 2 stages: In first stage, free radical polymerization of methyl methacrylate (MMA) was carried out to obtain syrup of polymethyl methacrylate. Polymerization parameters were determined in order to avoid autoacceleration effect and to get syrup which PMMA conversion avoids precipitation of ATH particles. In second stage, composite samples were manufactured using the obtained syrup, ATH and CMN particles and, then, they were tested in 
order to determine suitable parameters to get similar properties to Krion ${ }^{\circledR}$. It has been proved that syrup must have a MMA to PMMA conversion between $20 \%$ and $25 \%$ to avoid autoacceleration effect and precipitation of ATH particles. To obtain such syrup, polymerization must be carried out using $0,1 \%$ of benzoyl peroxide (PBO) y $0,2 \%$ of ethylene glycol dimercaptoacetate (EGDM) at $85^{\circ} \mathrm{C}$ during 90 minutes. Similarly, to get composites which properties are similar to Krion ${ }^{\circledR}$, the manufacturing parameters of a PMMA composite reinforced with ATH and CMN particles are: 40\% syrup / 60\% ATH / $\% \mathrm{CMN}$ variable according to the type of filler, $0,1 \%$ of additional $\mathrm{PBO}, 2,0 \%$ ethylene glycol dimethacrylate (EGDMA) y curing time 13 hours inside a furnace at $85^{\circ} \mathrm{C}$. Properties of the composite that has been manufactured under these conditions and only contains ATH filler are: flexural resistance of $53 \mathrm{MPa}$, flexural elastic modulus of $8054 \mathrm{MPa}$ and hardness of 91 HRM. Esthetic quality improves with the incorporation of CMN but mechanical properties decrease.

Keywords: solid surface, radical polymerization, polymethyl methacrylate, alumina trihydrate, natural mineral filler.

\section{INTRODUCCIÓN}

La polimerización en masa vía radicales libres es aplicada a nivel industrial en la producción de jarabes acrílicos, gracias a la cual se consigue, por ejemplo, un jarabe de polimetilmetacrilato, cuya elaboración está limitada por el fenómeno de autoaceleración [1]. Dichos jarabes pueden ser utilizados para la fabricación de materiales compuestos de matriz polimérica reforzados con partículas inorgánicas (como el trihidróxido de aluminio), los cuales se denominan comúnmente como materiales de superficie sólida (solid surface materials). En 1963, la compañía Dupont desarrolla el Corian ${ }^{\circledR}$ que es el primer material de superficie sólida obtenido a partir de la mezcla de un jarabe de polimetilmetacrilato con partículas de trihidróxido de aluminio [2-5].

Los materiales de superficie solida se caracterizan por presentar una apariencia similar a las piedras naturales, pero con baja densidad, y son utilizados como materiales de construcción en fachadas y diseño de interiores [6] por su capacidad para ser termoformados [7], su buena resistencia al fuego [8-9] y alta calidad estética [10]. Debido a sus buenas propiedades mecánicas estos materiales pueden ser utilizados para fabricar piezas de formas complicadas, las cuales no se podrían obtener a partir de grandes bloques de piedras naturales, tales como granito o mármol.

Muchos de los materiales de superficie solida comerciales están constituidos principalmente por polimetilmetacrilato (PMMA) y trihidróxido de aluminio (ATH), el cual además de ser usado como refuerzo, evita la propagación del fuego en este tipo de materiales [9]. En la literatura hay pocas investigaciones de materiales compuestos de PMMA/ATH, debido posiblemente a que estos materiales están patentados y sus formulaciones son celosamente guardadas por las empresas que los fabrican. En este sentido, toda la información sobre las formulaciones y pistas para fabricación de estos materiales provienen de las patentes caducas de DuPont.

Las investigaciones actuales de materiales compuestos de PMMA/ATH están relacionadas principalmente con desarrollo de modelos para predecir propiedades mecánicas, estudios de mecanismos de falla, reutilización de residuos generados en la fabricación de estos materiales y estudios de durabilidad.

Zhang [11] desarrolla modelos numéricos capaces de predecir las propiedades mecánicas de materiales compuestos reforzados con partículas, con aplicaciones específicas en materiales compuestos de PMMA/ATH. Los resultados teóricos fueron comparados con resultados experimentales obtenidos por Zhang en un estudio previo [12]. Análogamente, Gunel [13] utiliza un modelo de celda unitaria de partículas múltiples para estudiar el efecto de la temperatura y el contenido de partículas sobre las propiedades de materiales compuestos reforzados con partículas; luego utiliza los modelos propuestos para predecir las propiedades de materiales compuestos de PMMA/ATH.

Basaran [14] demuestra que la adhesión entre el refuerzo de ATH y la matriz de PMMA tiene una influencia significativa sobre las propiedades mecánicas. Por su parte, Nie [15] estudió el mecanismo de falla de materiales compuestos de PMMA/MMA en función de la adhesión entre la matriz y el refuerzo. Nie demostró que cuando adhesión entre el PMMA y ATH es alta, la fractura inicia en las aglomeraciones de las partículas de ATH presentes en el material; mientras que cuando la adhesión es baja, la fractura inicia cuando las aglomeraciones de ATH empiezan a separarse de la matriz. La importancia de estos estudios radica en la relación que existe entre la morfología de fractura y las propiedades mecánicas.

En otro contexto, Tušar [17-18] estudió la posibilidad de incorporar polvo de materiales compuestos de PMMA/ ATH como rellenos alternativos en la construcción de veredas. Se ha demostrado que el uso de estos rellenos alternativos mejora la adhesión entre los constituyentes de las mezclas que se elaboran para la construcción de veredas. Este estudio es pertinente, considerando que anualmente se generan 85 mil toneladas de residuos de materiales compuestos de PMMA/ ATH en forma de polvo que proviene de la fabricación de estos materiales [19].

Finalmente, Byrdy [16] sometió planchas de materiales compuestos de PMMA/ATH a envejecimiento acelerado bajo diversas condiciones ambientales tales como: alta temperatura, alta humedad relativa, ciclos de congelamiento y 
descongelamiento y radiación UV, con el propósito de verificar si estos pueden ser usados en ambientes exteriores bajo las condiciones climáticas de Europa Central.

También existen otros estudios de materiales compuestos de matriz de PMMA reforzados con partículas minerales diferentes al ATH. Por ejemplo, Alzarrug [20] utilizó refuerzos de nanopartículas de alúmina con el propósito de mejorar las propiedades mecánicas de materiales compuestos de PMMA; se ha encontrado una reducción del módulo elástico y aumento de la dureza de estos materiales comparado con un material polimérico sin partículas. Similarmente, Liu [21] encapsuló partículas de alúmina en PMMA mediante una polimerización en emulsión, logrando mejorar significativamente la estabilidad térmica y dispersión del refuerzo en los materiales compuestos elaborados. Asimismo, Ash [22] fabricó materiales compuestos de PMMA y nanopartículas de alúmina vía radicales libres, demostrando que una buena dispersión de las nanopartículas logra cambiar significativamente el comportamiento térmico y mecánico de un polímero termoplástico.

Se observa que las investigaciones recientes no profundizan en la fabricación de materiales compuestos de PMMA/ ATH y tampoco existen trabajos que impliquen el uso de cargas minerales naturales. En este sentido, el objetivo principal de esta investigación es desarrollar un material compuesto de propiedades similares al Krion ${ }^{\circledR}$, material comercial desarrollado por la empresa Systempool del grupo Porcelanosa, así como mejorar su aspecto estético utilizando cargas minerales naturales, tales como mica, cuarzo y marmolina. Para este propósito se fabricaron placas de materiales compuestos a partir de un jarabe de polimetilmetacrilato (PMMA), trihidróxido de aluminio (ATH) y cargas minerales naturales $(\mathrm{CMN})$, mediante moldeo por compresión. Estas placas fueron caracterizadas mediante ensayos de flexión (ASTM D790), dureza Rockwell (ASTM D785) y resistencia al desgaste (ASTM G65). Finalmente se compararon las propiedades obtenidas con las del Krion ${ }^{\circledR}$.

\section{MATERIALES Y MÉTODOS}

\subsection{Materiales y equipos}

Para la elaboración del jarabe se utilizaron los siguientes reactivos: metacrilato de metilo (MMA, estabilizado), peróxido de benzoilo (PBO), etilenglicol dimercapto acetato (EGDM); éstos fueron adquiridos de la empresa Merck y son de grado químicamente puro. El PBO fue secado a $35^{\circ} \mathrm{C}$ durante 5 horas para eliminar el $25 \%$ de humedad contenida. Asimismo, para la caracterización del jarabe se utilizaron los solventes: cloroformo y n-heptano de grado HPLC adquiridos de Merck y J.T. Baker, respectivamente.

Para la fabricación de los materiales compuestos se utilizaron los siguientes reactivos: peróxido de benzoilo (PBO) adicional, etilenglicol dimetacrilato (EGDMA), trihidróxido de aluminio (ATH) y cargas minerales naturales (CMN). El ATH, comercialmente denominado Onyx Elite ${ }^{\circledR} 255$, fue adquirido de la empresa Huber Engineered Materials y se caracteriza por tener 99,6 \% de $\mathrm{Al}(\mathrm{OH})_{3}$ y un tamaño de partícula promedio de $30 \mu \mathrm{m}$. Las $\mathrm{CMN}$ empleadas: mica (MI), marmolina (MA) y cuarzo (CU), que se muestran en la Figura 1, se adquirieron de la Compañía Minera Agregados Calcáreos S.A. del Perú. Los tamaños de las CMN utilizadas fueron: mica de $150 \mathrm{~mm}$ a 1,59 mm de tamaño, cuarzo de $63 \mathrm{~mm}$ a $105 \mathrm{~mm}$ y marmolina de $75 \mathrm{~mm}$ a $1,59 \mathrm{~mm}$.

Los experimentos de polimerización se realizaron en un reactor cerrado marca Parr, modelo 5112, que consta de un controlador Parr 4840, un vaso de vidrio de $1500 \mathrm{ml}$ de capacidad que soporta presiones y temperaturas máximas de 150 psi y $225^{\circ} \mathrm{C}$ que incluye sistemas de calentamiento y refrigeración. Los materiales compuestos se fabricaron en un molde de Alumold ${ }^{\circledR}$ de cavidad rectangular $\left(280 \times 75 \times 12,7 \mathrm{~mm}^{3}\right)$ con tapa.

Además se utilizó una prensa hidráulica manual de 20 toneladas y un horno eléctrico de $350{ }^{\circ} \mathrm{C}$. Los ensayos de flexión y dureza fueron realizados con una máquina de ensayo Zwick/Roell Z050 y un durómetro Rockwell 20021T, respectivamente. Los ensayos de desgaste fueron realizados con un equipo de diseño y fabricación propia según ASTM G65.

\subsection{Metodología experimental}

La fabricación de los materiales compuestos se llevó a cabo en dos etapas: 1) Polimerización parcial vía radicales libres del monómero MMA en el reactor para obtener un jarabe de PMMA y 2) Fabricación de placas de materiales compuestos a partir de la mezcla del jarabe de PMMA con partículas de ATH y CMN.

En la primera etapa, se estudiaron los parámetros del proceso de polimerización parcial del MMA, tales como: porcentaje de iniciador $(\% \mathrm{PBO})$, temperatura de reacción, tiempo de polimerización parcial y porcentaje de agente de 
transferencia de cadena (\%EGDM), con el propósito de obtener un jarabe con porcentaje de conversión y viscosidad adecuados para evitar la sedimentación de las partículas de ATH en la segunda etapa y el efecto de autoaceleración (efecto Trommsdorff) en el reactor [1].

En la segunda etapa, se estudiaron los parámetros del proceso de fabricación de materiales compuestos tales como: tiempo de curado $\left(\mathrm{t}_{\mathrm{c}}\right)$, porcentaje de trihidróxido de aluminio $(\% \mathrm{ATH})$ y cargas minerales naturales $(\% \mathrm{CMN})$, con el objetivo de lograr propiedades mecánicas similares al producto comercial de referencia denominado Krion®. Para comparar las propiedades de los materiales fabricados con las del comercial, se realizaron ensayos de flexión según ASTM D790 y dureza Rockwell según ASTM D785 y, complementariamente, ensayos de resistencia al desgaste según ASTM G65.

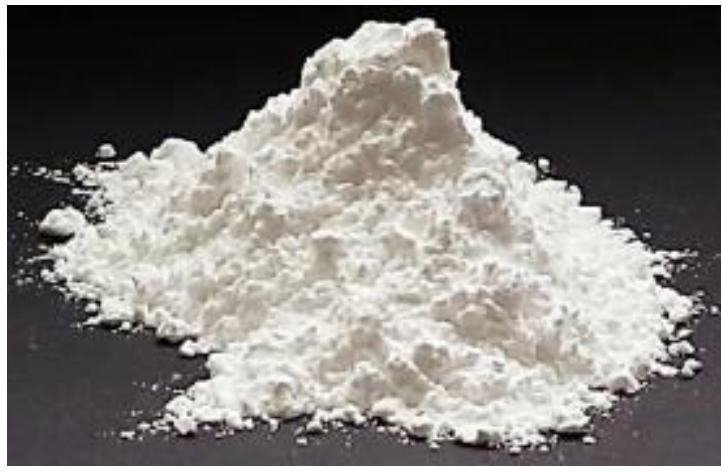

(a) Trihidróxido de aluminio (ATH)

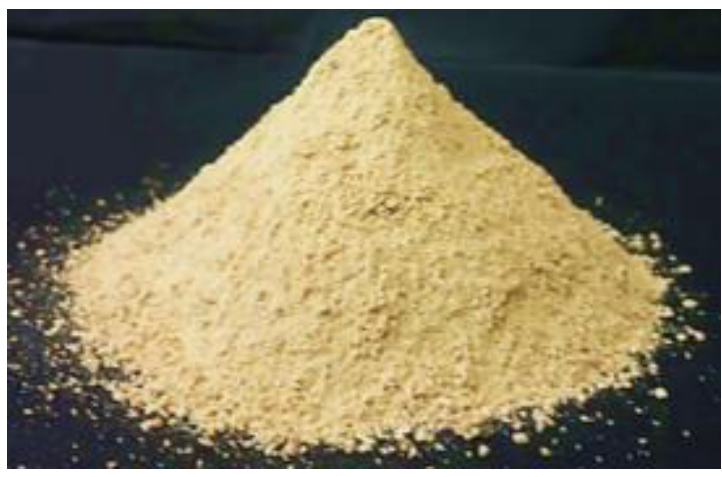

(c) Marmolina (MA)

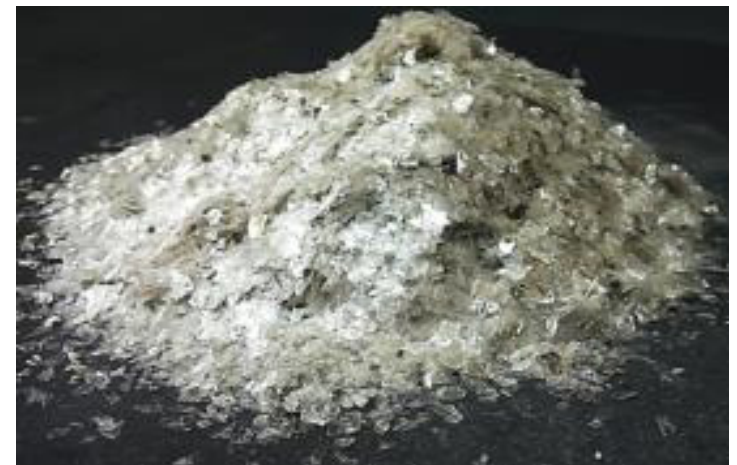

(b) Mica (MI)

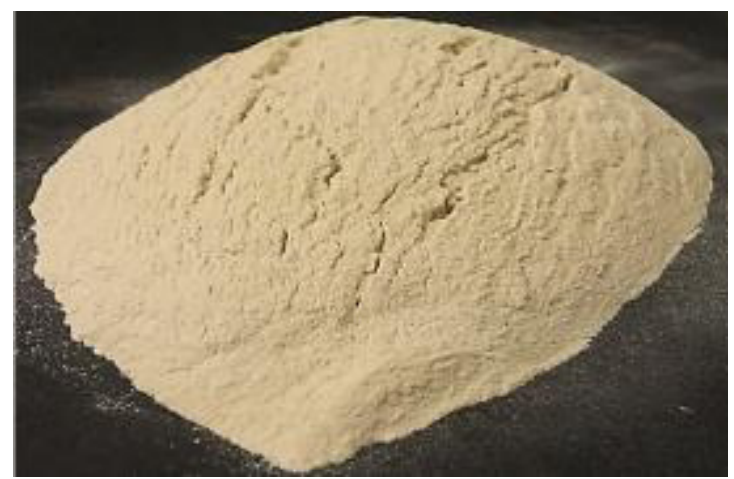

(d) Cuarzo (CU)

Figura 1: Carga mineral retardante de fuego (a), cargas minerales de origen natural (b), (c) y (d)

\subsection{Procedimiento experimental}

\subsubsection{Etapa 1: Elaboración del jarabe de PMMA}

En la elaboración de un jarabe, inicialmente se deben definir los parámetros de polimerización. Así, por ejemplo, un jarabe típico se elabora con: $0,1 \% \mathrm{PBO}, 0,2 \% \mathrm{EGDM}, 85^{\circ} \mathrm{C}$ de temperatura de reacción y 115 minutos de tiempo de polimerización parcial. Si se desea obtener $250 \mathrm{~g}$ de jarabe, inicialmente se deben disolver 0,3304 g de PBO y 0,6608 g de EGDM en $350 \mathrm{ml}$ de MMA (330,4 g) en el vaso del reactor; cabe indicar que las masas de PBO y EGDM se calculan en relación a la masa inicial de MMA. Luego, en el reactor, dicha solución se agita a una velocidad de 350 RPM y se calienta a una temperatura constante de $85^{\circ} \mathrm{C}$ durante 115 minutos medidos desde el inicio del calentamiento a temperatura ambiente. Como la solución requiere aproximadamente 25 minutos para llegar a $85{ }^{\circ} \mathrm{C}$, se ha visto conveniente definir el tiempo de polimerización parcial como la diferencia: $(115$ - 25) $\min =90 \mathrm{~min}$. Así mismo, considerando que la reacción de polimerización es exotérmica, para mantener la temperatura de reacción en $85^{\circ} \mathrm{C}$, se debe activar el sistema de refrigeración del reactor cada vez que la temperatura tiende a variar.

Después de la reacción se obtiene un jarabe de PMMA, el cual debe ser caracterizado para determinar el porcentaje de conversión de MMA en PMMA (\%PMMA), su viscosidad inherente () y su peso molecular (M). Para determinar el porcentaje de conversión, se toman muestras del jarabe elaborado (10 mg a $40 \mathrm{mg}$ ) que luego de ser pesadas se disuelven en cloroformo $(20 \mathrm{ml})$. La solución obtenida se añade lentamente en n-heptano $(140 \mathrm{ml})$ para conseguir la precipitación del PMMA. Luego, la solución de n-heptano es filtrada con el fin de separar el 
PMMA que, posteriormente, debe ser secado a $40{ }^{\circ} \mathrm{C}$ el tiempo necesario hasta que la variación de peso sea despreciable. Finalmente, el \%PMMA se calcula con la ecuación 1.

$$
\% P M M A=\frac{\text { masa PMMA seco }}{\text { masa de muestra de jarabe }} \times 100 \%
$$

Por otro lado, el peso molecular del PMMA se calcula a partir de su viscosidad inherente $\left(\eta_{\text {inh }}\right)$. Para determinar dicha viscosidad, se disuelve PMMA seco $(0,25 \mathrm{~g})$ en cloroformo $(50 \mathrm{ml})$ y dicha solución se hace pasar por un viscosímetro de Ubbelohde a $20^{\circ} \mathrm{C}$; registrándose el tiempo (t) que la solución tarda en pasar a través de las marcas establecidas en el viscosímetro. Luego, se calcula la viscosidad inherente utilizando la ecuación 2, donde " $\mathrm{t}_{0}$ " es el tiempo que el cloroformo puro tarda en pasar por las marcas del viscosímetro y "C" ( $\mathrm{g} / \mathrm{dl})$ es la concentración del PMMA seco disuelto en el cloroformo a $20^{\circ} \mathrm{C}$ [23]. Finalmente, el peso molecular ( $\mathrm{g} / \mathrm{mol}$ ) se calcula con la ecuación 3 (Mark-Houwink-Sakurada [24]), donde los las constantes: $\mathrm{K}=0,00485 \mathrm{ml} / \mathrm{g}$ y a $=0,8$ son válidas para el PMMA disuelto en cloroformo a $20^{\circ} \mathrm{C}$.

$$
\begin{aligned}
& \eta_{\text {inh }}=\ln \left(t / t_{0}\right) \times C^{-1} \\
& M=\left(\eta_{\text {inh }} \times K^{-1}\right)^{1 / a}
\end{aligned}
$$

\subsubsection{Etapa 2: Fabricación del material compuesto}

Análogamente, para fabricar un material compuesto previamente se deben definir los parámetros de fabricación. Por ejemplo, para un material compuesto típico de $40 \%$ jarabe / 60\% ATH se utiliza: 0,1\% de PBO, 2,0\% de EGDMA, 3,0\% de $\mathrm{CMN}$ y una temperatura y tiempo de curado de $85^{\circ} \mathrm{C}$ y 6 horas respectivamente. Si se desea obtener una placa rectangular de $280 \times 75 \times 12,7 \mathrm{~mm}^{3}$, se requieren $250 \mathrm{~g}$ de jarabe. Inicialmente dicha cantidad de jarabe se mezcla con $0,25 \mathrm{~g}$ de PBO y 5,0 g de EGDMA, luego, se agregan y mezclan 7,50 g de CMN y $375 \mathrm{~g}$ de ATH, en ese orden; cabe aclarar que las masas de PBO, EGDMA y CMN se calculan en relación a la masa inicial de jarabe y la masa de ATH se calcula considerando la relación en peso jarabe/ATH que en este caso es 40/60. Seguidamente se vierten en el molde rectangular $465 \mathrm{~g}$ de la mezcla obtenida y se tapa ejerciendo una fuerza de 20 toneladas, con el propósito de remover las burbujas de aire que fueron atrapadas durante el mezclado y obtener placas de buen acabado superficial. Finalmente, para realizar el curado, el molde se lleva a un horno eléctrico a $85{ }^{\circ} \mathrm{C}$ durante 6 horas. El tiempo de curado inicia una vez que el molde ha alcanzado los 85 ${ }^{\circ} \mathrm{C}$, lo cual puede tardar aproximadamente 45 minutos.

Luego del desmolde, se obtiene una placa rectangular que debe ser caracterizada para determinar sus propiedades. Para ello, a partir de dicha placa se mecanizan tres probetas para los ensayos de flexión, dureza y desgaste respectivamente. Es preciso explicar que el ensayo de desgaste permite evaluar la resistencia al desgaste de forma indirecta cuantificando la pérdida de masa durante dicho ensayo.

\section{RESULTADOS Y DISCUSIÓN}

\subsection{Etapa 1: Elaboración de jarabe (PMMA/MMA)}

\subsubsection{Estudio de la temperatura de reacción}

Se estudió el efecto de la temperatura de reacción sobre el tiempo de polimerización parcial y el porcentaje de conversión de MMA a PMMA en varios jarabes elaborados con $0,1 \%$ de PBO, de tal forma que no se produzca el fenómeno de autoaceleración (efecto Trommsdorff) en el reactor.

La Figura 2 muestra la variación del tiempo de reacción y \% PMMA en función de la temperatura de reacción usando $0,1 \%$ de $\mathrm{PBO}$ en peso respecto del monómero. Como era de esperarse, el tiempo de reacción disminuye y la conversión de MMA a PMMA (\%PMMA) aumenta con el incremento de la temperatura de reacción [25]. Asimismo, se deduce que las temperaturas adecuadas de polimerización son $80^{\circ} \mathrm{C}$ y $85^{\circ} \mathrm{C}$, ya que a estas temperaturas se obtuvo porcentajes de conversión del MMA a PMMA en el rango de $17 \%$ a $19 \%$. A $70{ }^{\circ} \mathrm{C}$ la conversión es mínima debido a la muy baja cantidad de radicales libres generados por el iniciador.

Entonces, en la primera etapa, con el propósito de obtener una mayor conversión en un tiempo menor, se seleccionó una temperatura de reacción de $85^{\circ} \mathrm{C}$. Con esta temperatura se logró alrededor de $19 \%$ en peso de conversión de MMA en PMMA en aproximadamente 90 minutos, con un buen control de temperatura y sin autoaceleración. 


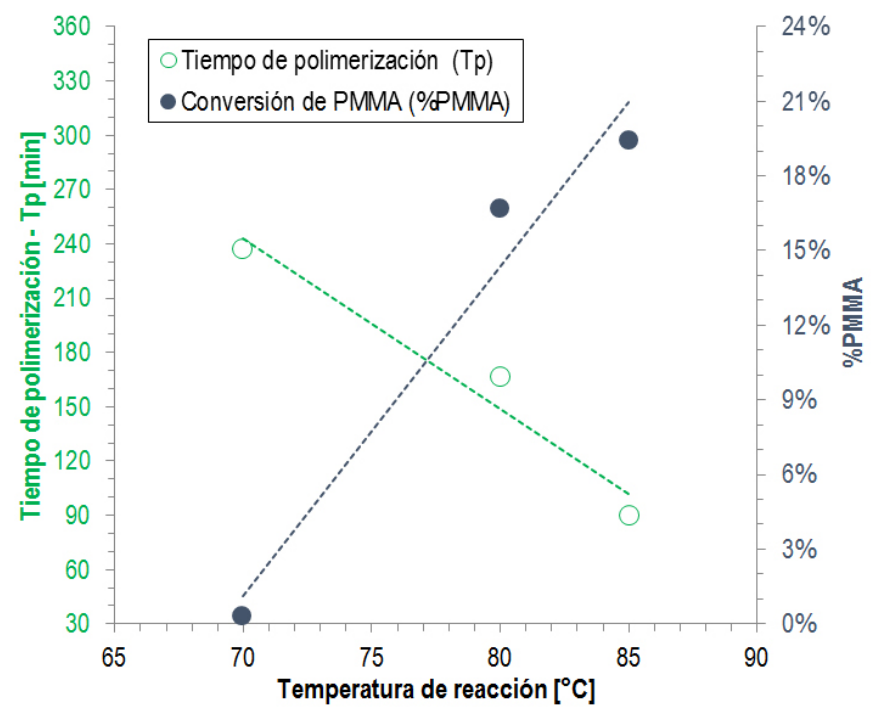

Figura 2: Tiempo de polimerización y \%PMMA en función de la temperatura de reacción con $0,1 \%$ PBO.

\subsubsection{Estudio del contenido de agente de transferencia de cadena}

Análogamente, se estudió la influencia del agente de transferencia de cadena etilenglicol dimercapto acetato (EGDM) sobre la conversión y el peso molecular del PMMA, en distintos jarabes elaborados con los parámetros definidos previamente: $0,1 \%$ en peso de $\mathrm{PBO}$, temperatura y tiempo de reacción de $85^{\circ} \mathrm{C}$ y 90 minutos respectivamente.

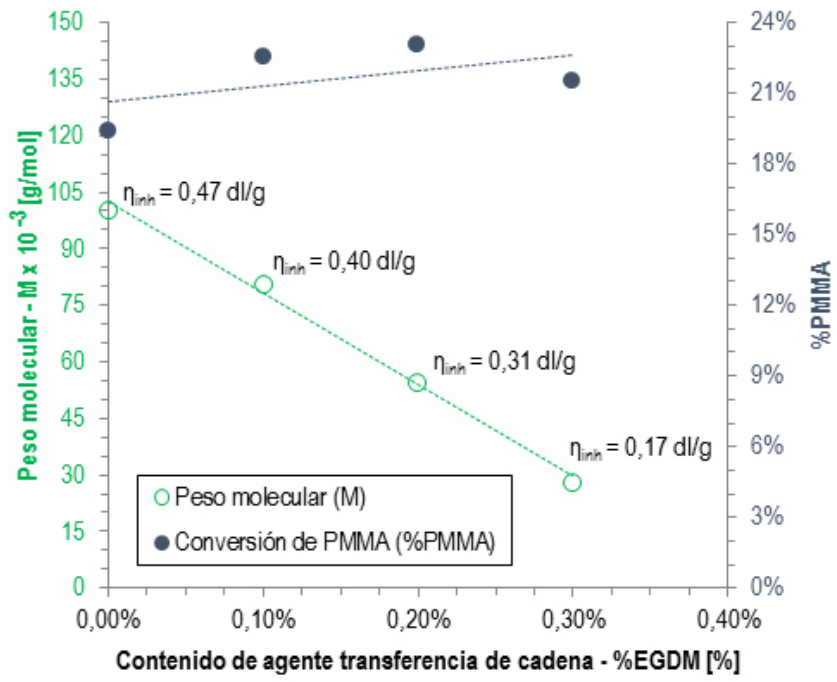

Figura 3: Peso molecular y conversión de PMMA en función del \%EGDM, con 0,1\% PBO a $85{ }^{\circ} \mathrm{C}$ y 90 min.

La Figura 3 muestra la variación del peso molecular (M en g/mol) y el porcentaje de conversión de MMA en PMMA (\%PMMA), en función del \%EGDM. En esta figura se observa que mientras \%PMMA prácticamente se mantiene constante en el rango de 20 a $25 \%$, el peso molecular disminuye considerablemente con el incremento del \%EGDM, a tal punto que con $0,3 \%$ EGDM la viscosidad del jarabe es tan baja $(0,17 \mathrm{dl} / \mathrm{g})$ que permite la sedimentación de las partículas de ATH en los materiales compuestos. Para evitar la sedimentación de ATH, Duggins et al [3,5] determinaron que la viscosidad debe estar en el rango: $0,30-0,55 \mathrm{dl} / \mathrm{g}$. Se ha observado que placas fabricadas con jarabes que tienen PMMA de bajo peso molecular (viscosidad menor a $0,17 \mathrm{dl} / \mathrm{g}$ ) presentan sedimentación de las partículas de ATH, lo cual implica la presencia de 2 fases bien diferenciadas a lo largo del espesor de dichas placas: una de PMMA puro y otra de PMMA y partículas de ATH.

Por tanto, las condiciones para obtener un jarabe con un contenido de 20 a $25 \%$ de PMMA y una viscosidad inherente alrededor de $0,31 \mathrm{dl} / \mathrm{g}(55000 \mathrm{~g} / \mathrm{mol})$, adecuada para evitar el fenómeno de autoaceleración en el reactor e impedir la sedimentación de las partículas de ATH, son: 0,1\% PBO y 0,2\% EGDM en peso, a una temperatura y tiempo de reacción de $85^{\circ} \mathrm{C}$ y 90 minutos respectivamente. 
De forma análoga al resultado anterior, con el objetivo de determinar la cinética de conversión del PMMA, se han elaborado varios jarabes con los parámetros determinados previamente: $0,1 \% \mathrm{PBO}, 0,2 \% \mathrm{EGDM}$ y $85^{\circ} \mathrm{C}$ de temperatura de reacción, variando el tiempo de polimerización (Figura 4). Como era de esperarse, en la Figura 4, se observa que el \%PMMA aumenta con el tiempo de polimerización. Asimismo, como en el caso anterior, se ha conseguido un jarabe con 23,6 \% PMMA en 90 min, corroborando dicho resultado. También se observa que la conversión de PMMA empieza a acelerarse rápidamente a partir de los 100 minutos, lo cual podría señalar el inicio del fenómeno de autoaceleración.

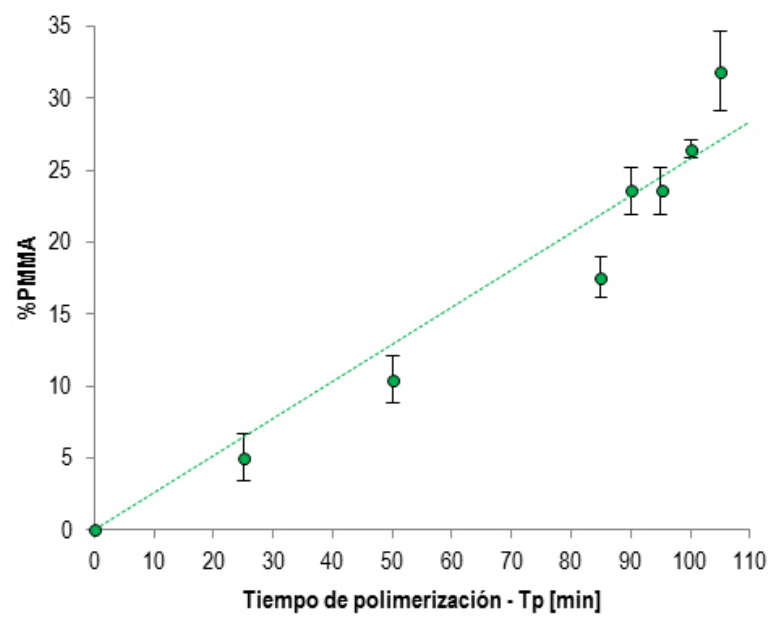

Figura 4: Cinética de conversión del PMMA con 0,1\% PBO, 0,2\% EGDM, $85{ }^{\circ} \mathrm{C}$ y $90 \mathrm{~min}$.

\subsection{Etapa 2: Fabricación del material compuesto}

\subsubsection{Estudio de la influencia del tiempo de curado (Tc)}

Con el objetivo de estudiar la influencia del tiempo de curado sobre las propiedades mecánicas, se fabricaron varios materiales compuestos mezclando el jarabe definido en la etapa 1 para obtener de $20 \%$ a $25 \%$ PMMA, $0,1 \%$ de PBO adicional, $2,0 \%$ de EGDMA y $60 \%$ de ATH, para luego someterlos a $85^{\circ} \mathrm{C}$ de temperatura y distintos tiempos de curado.

En la Figura 5, las líneas de tendencia indican que la resistencia a la flexión y el módulo elástico de estos materiales aumentan con el tiempo de curado, aunque con poca pendiente. Asimismo, utilizando dichas líneas de tendencia, se estima que se requerirían 9,6 h ó 16,6 h de tiempo de curado para conseguir un material compuesto cuya resistencia o módulo, respectivamente, sean iguales a la del producto comercial $\mathrm{Krion}{ }^{\circledR}\left(\mathrm{R}_{\mathrm{f}}=63 \mathrm{MPa}\right.$ y $\left.\mathrm{E}_{\mathrm{f}}=8948 \mathrm{MPa}\right)$. A partir de estos tiempos estimados, se decidió fijar un tiempo de curado medio de $13 \mathrm{~h}$ para la fabricación de los materiales compuestos necesarios en los siguientes estudios. Es preciso indicar que el proceso de curado, a $85^{\circ} \mathrm{C}$, se realizó en dos etapas: (i) 1,0 hora de tiempo de curado dentro del molde y (ii) 12,0 horas de tiempo de curado fuera del molde.

Por otro lado, en la misma Figura 5, se puede ver que se ha logrado fabricar placas solidas de material compuesto con 0,5 horas de tiempo de curado, con lo cual se deduce que el tiempo de solidificación puede ser aún menor. Se supone que las tasas de crecimiento tanto de la resistencia a la flexión $(2,34 \mathrm{MPa} / \mathrm{h})$ como del módulo elástico en flexión $(86,3 \mathrm{MPa} / \mathrm{h})$ son bajas, precisamente porque el material cambia de estado líquido viscoso a sólido en corto tiempo y, en consecuencia, se dificulta el reticulado del PMMA posiblemente porque la difusión del EGDMA (reticulador) es más lenta al realizarse en estado sólido. Por tanto, tiempos de curado cortos implican un bajo grado de reticulado y pobres propiedades mecánicas, mientras que, tiempos de curado largos permiten alcanzar un mejor grado de reticulado y mejores propiedades mecánicas. 


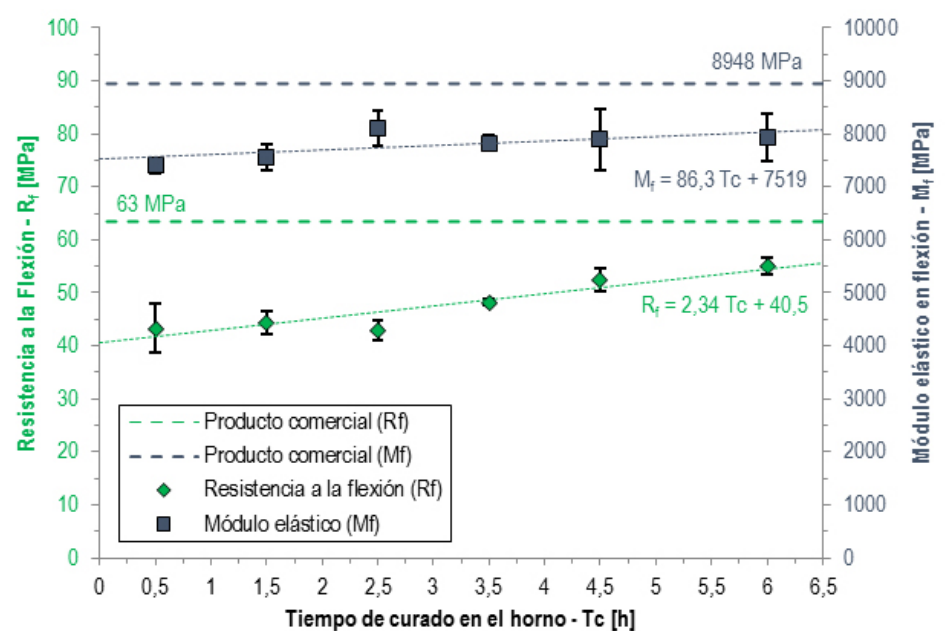

Figura 5: Resistencia y módulo elástico en flexión en función del tiempo de curado.

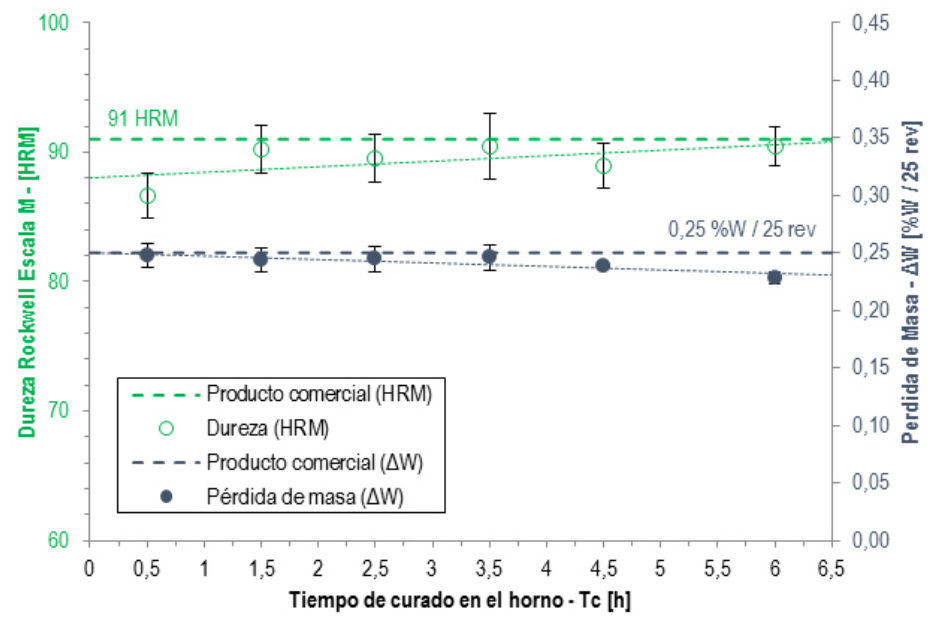

Figura 6: Dureza y resistencia al desgaste en función del tiempo de curado.

Para corroborar los resultados de los ensayos de flexión, los materiales compuestos fabricados fueron sometidos a ensayos de dureza y resistencia al desgaste (pérdida de masa) como se observa en la Figura 6. Se puede apreciar que en el caso de la dureza, la tendencia creciente es similar a las tendencias de la resistencia y el módulo, lo cual es coherente; mientras que en el caso de la pérdida de masa, durante el ensayo de la resistencia al desgaste de dichos materiales, se observa que ésta disminuye con el incremento del tiempo de curado o, dicho de otra manera, que la resistencia al desgaste aumenta con el tiempo de curado, siendo éste también coherente con los resultados anteriores. Cabe resaltar que la dureza del material fabricado con 6 horas de tiempo de curado es prácticamente igual a la dureza del producto comercial Krion ${ }^{\circledR}$ y que, en todos los casos, la pérdida de masa de dichos materiales son menores a la del producto comercial o de mejor resistencia al desgaste.

\subsubsection{Estudio del contenido de trihidróxido de aluminio (\% ATH)}

Análogamente, con el propósito de estudiar la influencia del contenido de ATH sobre las propiedades mecánicas, se fabricaron varios materiales compuestos mezclando el jarabe definido en la etapa 1 para obtener de $20 \%$ a $25 \%$ PMMA, $0,1 \%$ de $\mathrm{PBO}$ adicional, 2,0\% de EGDMA y distintos contenidos de ATH, para luego someterlo a un proceso de curado en molde a $85^{\circ} \mathrm{C}$ durante 13 horas. 

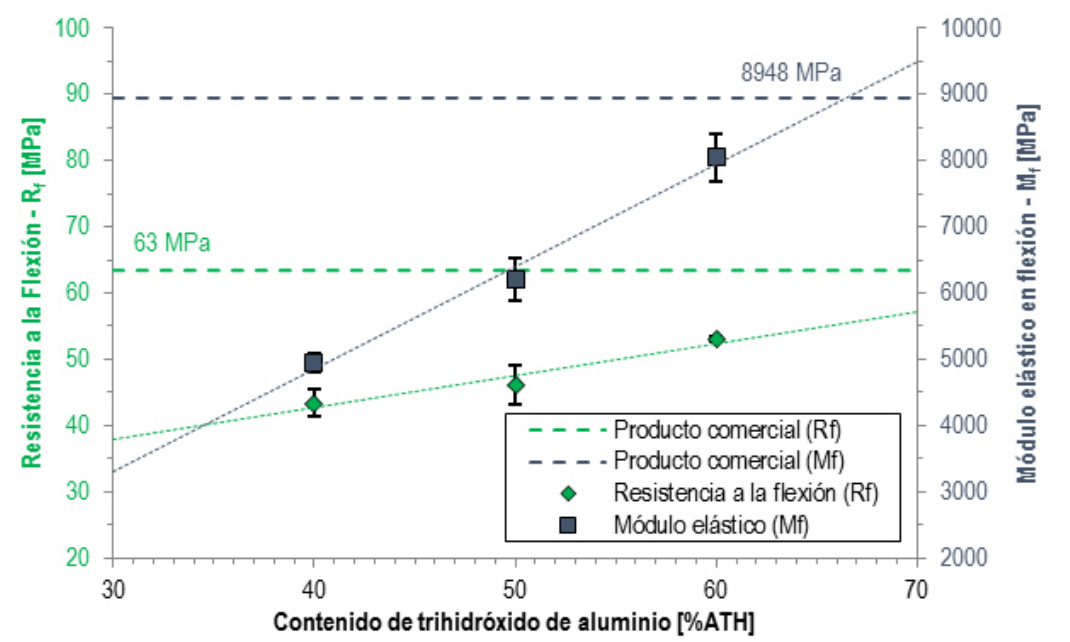

Figura 7: Resistencia y módulo elástico en flexión en función del \%ATH.

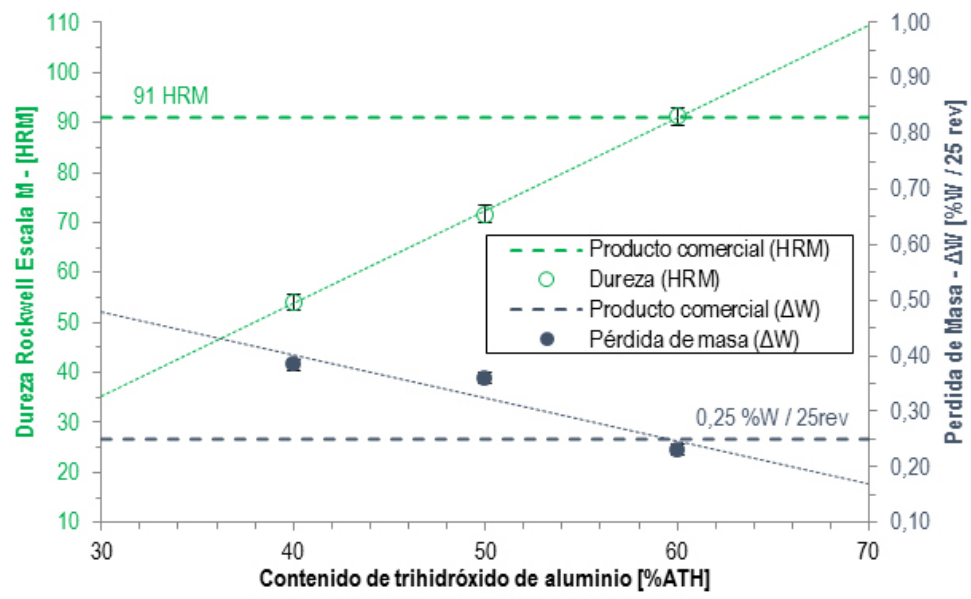

Figura 8: Dureza y resistencia al desgaste en función del \%ATH.

En la Figura 7 se observa que tanto la resistencia como el módulo elástico en flexión incrementan con el contenido de ATH y, según las líneas de tendencia correspondientes, estas propiedades pueden seguir en aumento más allá de lo demostrado experimentalmente en este trabajo. Sin embargo, cuando el contenido de partículas es muy elevado, dichas propiedades posiblemente estén limitadas por la generación de aglomeraciones macroscópicas de ATH que la matriz no es capaz de dispersar, en tanto que la mezcla es más difícil. Entonces, existe la posibilidad de encontrar cierto contenido de ATH que maximice las propiedades mecánicas de los materiales compuestos obtenidas por moldeo. Los resultados (Figura 7) muestran que el material con $60 \%$ de ATH tiene la más alta resistencia (53 MPa) y módulo elástico (8054 MPa) encontrados aunque dichos valores aún están por debajo del material comercial de referencia.

En coherencia con la resistencia y el modulo elástico en flexión de los mismos materiales, en la Figura 8 se observa que la dureza y la resistencia al desgaste (disminuye la pérdida de masa) también incrementan con el contenido de ATH; aunque a diferencia de aquellos, con $60 \%$ de ATH, se logra igualar la dureza y la resistencia al desgaste del producto comercial Krion ${ }^{\circledR}(0,25 \% \mathrm{~W} / 25 \mathrm{rev})$ aun cuando el \%ATH es menor. 


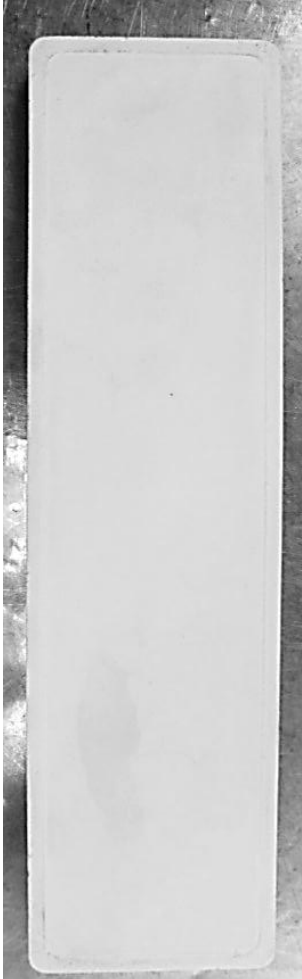

(a)

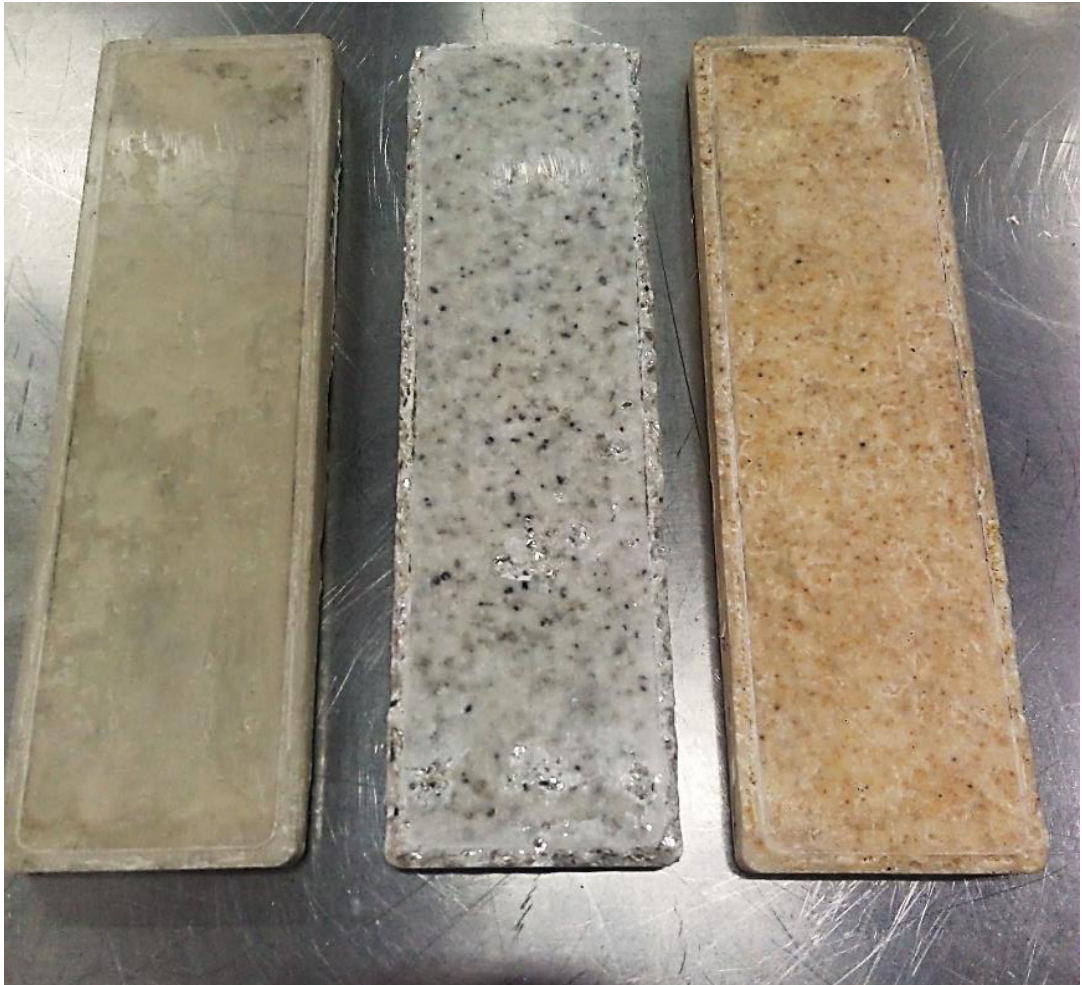

(b) (c)

Figura 9: Materiales compuestos con 60\% ATH (a) y cargas minerales naturales: cuarzo (b), mica (c), marmolina (d)

\subsubsection{Estudio del contenido de cargas minerales naturales (CMN)}

Con el fin de mejorar la apariencia estética de los materiales compuestos fabricados solo con ATH, se han incorporado además diferentes tipos de cargas minerales naturales: mica, marmolina y cuarzo. Estos materiales se fabricaron utilizando los parámetros definidos en los estudios previos para obtener placas con propiedades similares al producto comercial Krion®: jarabe con porcentaje de conversión de PMMA entre 20\% y 25\%, 0,1\% de PBO adicional, 2,0\% de EGDMA, 60\% de ATH, $3,0 \%$ de $\mathrm{CMN}, 85^{\circ} \mathrm{C}$ y 13 horas de temperatura y tiempo de curado respectivamente.

Como primer resultado, se observa que cada tipo CMN cambia particularmente la apariencia estética de los materiales compuestos fabricados solo con ATH, lo que permite diferenciarlos visualmente entre sí como se muestra en la Figura 9. Sin embargo, se debe destacar que en todos los casos, la apariencia física modificada por las partículas le proporciona al material compuesto una buena calidad estética. Cabe aclarar que el material denominado ATH $60 \%$ y el producto comercial Krion ${ }^{\circledR}$ contienen únicamente ATH, mientras que los otros materiales contienen ATH y además un tipo de CMN.

Las Figuras 10, 11 y 12 muestran los resultados de los ensayos de flexión, dureza y resistencia al desgaste. En la Figura 10 se observa que el material con $60 \%$ ATH tiene la mayor resistencia a la flexión (55 MPa) que los materiales elaborados con cuarzo (43 MPa), marmolina (39 MPa) y mica (35 MPa), lo que demuestra que dicha propiedad varía según el tipo de CMN. Posiblemente una de las principales razones se debe a las diferencias en la adhesión con la matriz de PMMA. Así, por ejemplo, al observar la zona de fractura de los materiales compuestos, se constata que las hojuelas de mica tienen una adhesión más débil con la matriz, puesto que tienden a desprenderse fácilmente en comparación con las partículas de marmolina y cuarzo, corroborando su menor resistencia a la flexión.

Por otro lado, el tamaño y la forma de las partículas de las CMN parece tener una influencia determinante sobre las propiedades del material compuesto. Una de las posibles razones para que el cuarzo tenga mejor resistencia que los otros materiales fabricados con CMN se debe a que las partículas equiaxiales de cuarzo (de $63 \mathrm{~mm}$ a $105 \mathrm{~mm}$ ) son similares en forma y tamaño a las partículas de ATH (tamaño promedio de $30 \mu \mathrm{m}$ ) y, al mismo tiempo, mucho menores en tamaño que las partículas equiaxiales de marmolina $(75 \mu \mathrm{m}-1,59 \mathrm{~mm})$; por tanto, como se evidencia, las partículas de cuarzo no precipitan y el material resulta ser más homogéneo en comparación con la marmolina o la mica cuyas partículas tienden a precipitar. Similarmente, respecto a la forma de las partículas, al parecer las hojuelas o láminas delgadas de mica con una deficiente adhesión a la matriz generan concentradores de tensión más críticos que las partículas equiaxiales de cuarzo o marmolina $\mathrm{y}$, consecuentemente, estos últimos fallan con esfuerzos más altos. 


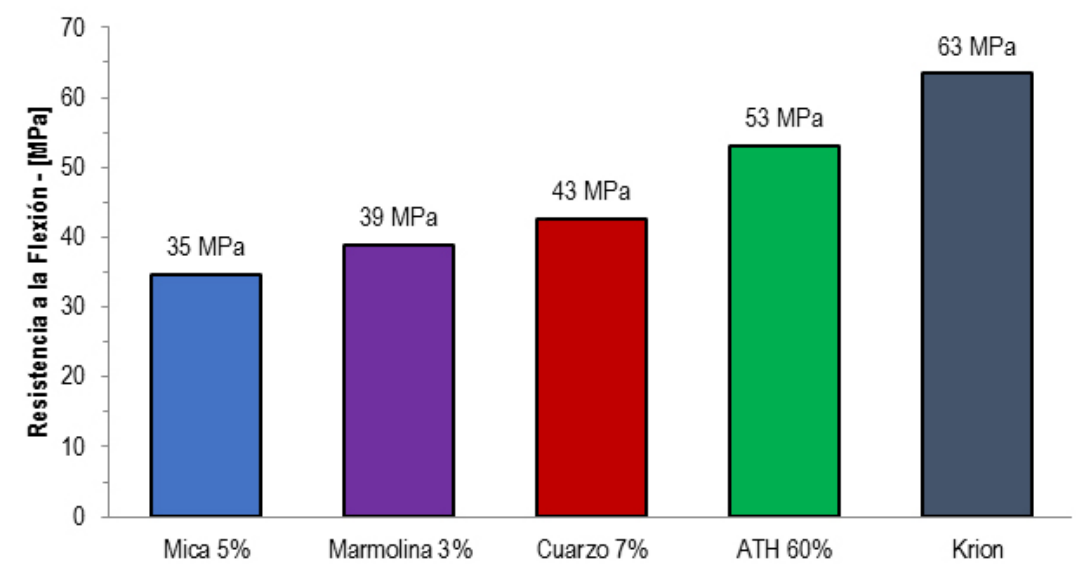

Figura 10: Resistencia a la flexión de materiales compuestos con ATH y ATH/CMN

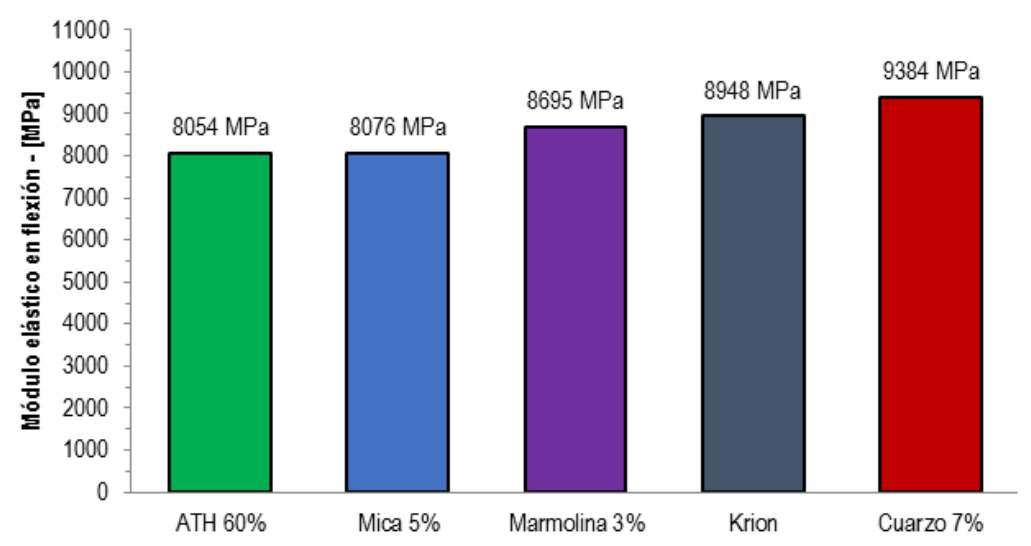

Figura 11: Módulo elástico en flexión de materiales compuestos con ATH y ATH/CMN

En la Figura 11 se observa que el material con $60 \%$ de ATH tiene un módulo elástico de $8054 \mathrm{MPa}$, que es menor que el módulo de los materiales elaborados con mica (8076 MPa), marmolina (8695 MPa) y cuarzo (9384 MPa), este último es el único que supera al Krion ${ }^{\circledR}(8948 \mathrm{MPa})$. Como era de esperar, el módulo elástico aumenta al añadir CMN y la mejora es más eficaz con la marmolina y el cuarzo que con la mica, posiblemente por las razones mencionadas en el caso de la resistencia a la flexión.

Los resultados de dureza observados en la Figura 12 corroboran los resultados de la resistencia a la flexión, al ser comparativamente similares: el material con $60 \%$ de ATH tiene una dureza mayor (91 HRM) que las durezas de los materiales elaborados con cuarzo (87 HRM), marmolina (85 HRM) y mica (79 HRM).

Finalmente, haciendo un compromiso entre la calidad estética y las propiedades, los mejores resultados fueron obtenidos con la incorporación de cuarzo en el material compuesto, el cual proporcionó una buena calidad estética (Figura 9) aun cuando no supero las propiedades mecánicas del material de referencia Krion ${ }^{\circledR}$ (Figuras 10, 11 y 12).

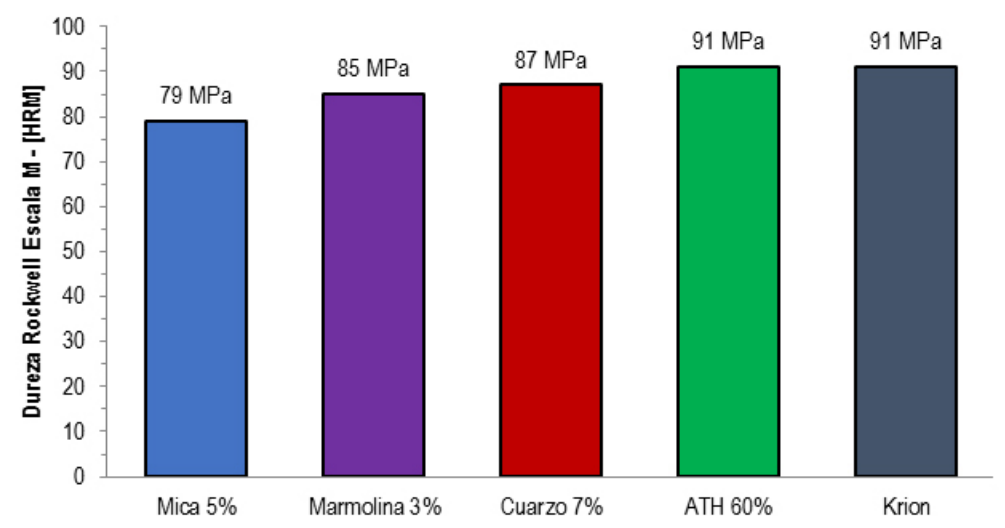

Figura 12: Dureza de materiales compuestos con ATH y ATH/CMN 


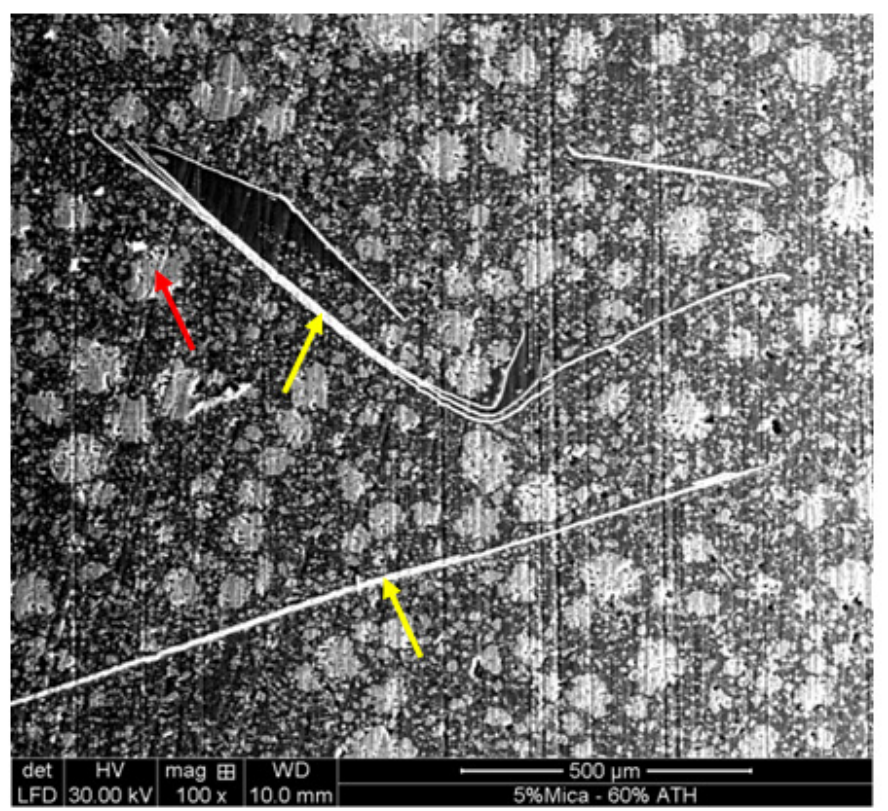

Figura 13: Distribución de las partículas de ATH (rojo) y hojuelas de mica (amarillo)

\subsubsection{Fotografías SEM de la zona de fractura de los materiales compuestos con cargas minerales}

La figura 13 muestra la distribución de las partículas de ATH y mica en un material compuesto de PMMA/ATH con 5\% de mica. Se observa que las partículas de ATH tienen forma y tamaño parecidos y están bien distribuidas a lo largo de la matriz de PMMA. En contraste, las hojuelas de mica, que tienen forma plana y tamaños más grandes que las partículas de ATH, no tienen una distribución homogénea ya que su tamaño y forma le impiden orientarse en cualquier posición. Asimismo, la figura 14 muestra la zona de fractura en flexión del material compuesto de PMMA/ATH con 5\% de mica. Se observa que existe falta de adherencia entre las hojuelas de mica y la matriz de PMMA, lo que origina que dicho material tenga bajas propiedades mecánicas en comparación con un material compuesto de PMMA/ATH sin cargas minerales adicionales (Figuras 10 y 12).

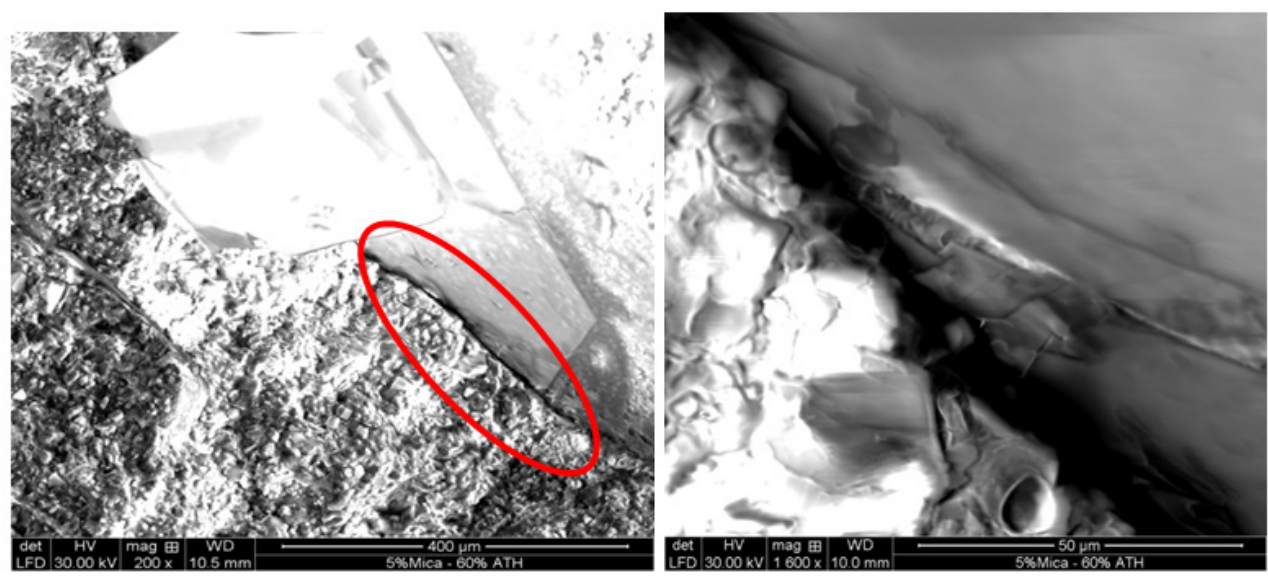

Figura 14: Material compuesto de PMMA/ATH con 5\% Mica - Interfaz entre matriz y hojuela de mica 


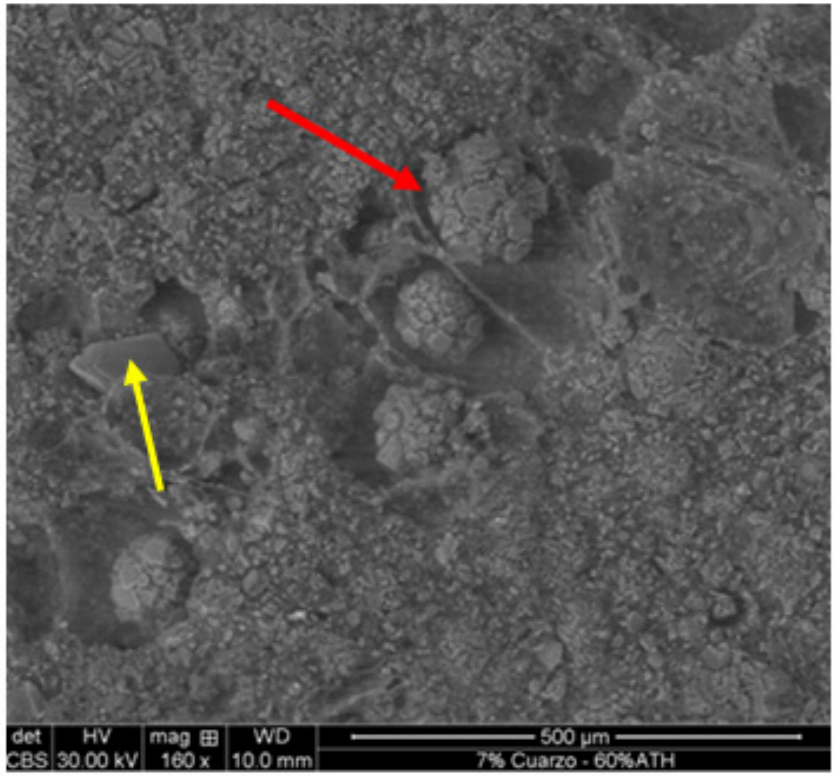

Figura 15: Zona de fractura de un material compuesto de PMMA/ATH (rojo) con 7\% de cuarzo (amarillo)

Por otro lado, la figura 15 muestra la zona de fractura de un material compuesto de PMMA/ATH con 7\% de cuarzo sometido a flexión. En la figura se observa que la matriz de PMMA se ha deformado alrededor de las partículas de ATH, con lo cual es posible concluir que no hay una buena adherencia entre las partículas de ATH y la matriz de PMMA. En coherencia con lo señalado por Nie et al [15], la fractura del material habría iniciado con la separación de las partículas de ATH de la matriz de PMMA, lo cual es una señal de una baja fuerza de adhesión entre el refuerzo y la matriz (ausencia de transferencia de carga entre matriz y refuerzo). Puesto que el producto comercial presenta mejores propiedades que los materiales fabricados en este estudio, es probable que para su fabricación se hayan utilizado tratamientos superficiales para mejorar la adhesión interfacial entre el PMMA y ATH, lo cual justificaría razonablemente que presente mejores propiedades mecánicas.

\section{CONCLUSIONES}

Se ha desarrollado un material compuesto de propiedades similares al Krion ${ }^{\circledR}$. Este material ha sido fabricado con $40 \%$ de jarabe de polimetilmetacrilato (PMMA), 60\% de trihidróxido de aluminio (ATH) y cargas minerales naturales (CMN) de contenido variable según el tipo de carga. Se han definido los parámetros de polimerización para la elaboración del jarabe, los parámetros de fabricación de los materiales compuestos con solo ATH y los parámetros de fabricación de los materiales compuestos con CMN adicionales.

El jarabe de PMMA, elaborado mediante polimerización parcial vía radicales libres, tiene un porcentaje de conversión que varía entre $20 \%$ y $25 \%$ en peso de PMMA, un peso molecular de alrededor de $55000 \mathrm{~g} / \mathrm{mol}$ correspondiente a una viscosidad inherente en el rango de 0,30 a $0,55 \mathrm{dl} / \mathrm{g}$, para evitar el fenómeno de autoaceleración en el reactor y, al mismo tiempo, la sedimentación de las partículas de ATH en el material compuesto. Los parámetros de dicha polimerización parcial son: $0,1 \%$ en peso de peróxido de benzoilo (PBO), $0,2 \%$ en peso de etilenglicol dimercaptoacetato(EGDM), $85^{\circ} \mathrm{C}$ temperatura de reacción y 90 minutos de tiempo de polimerización parcial.

Los parámetros para la fabricación de un material compuesto de matriz acrílica reforzado con partículas de ATH y de propiedades similares la Krion ${ }^{\circledR}$ son: $40 \%$ jarabe / $60 \%$ ATH, $0,1 \%$ en peso de $\mathrm{PBO}$ adicional, $2,0 \%$ en peso de etilenglicol dimetacrilato (EGDMA) y 13 horas de curado en un horno a $85^{\circ} \mathrm{C}$. Las propiedades del material compuesto fabricado bajo estas condiciones son: $53 \mathrm{MPa}$ de resistencia a flexión, $8054 \mathrm{MPa}$ de módulo elástico en flexión y 91 HRM de dureza.

Los parámetros para la fabricación de un material compuesto de matriz acrílica reforzado con partículas de ATH y cargas minerales naturales (CMN) son: 40\% jarabe / 60\% ATH / \%CMN variable según el tipo de carga, $0,1 \%$ en peso de PBO adicional, 2,0\% en peso de etilenglicol dimetacrilato (EGDMA) y 13 horas de curado en un horno a $85^{\circ} \mathrm{C}$. En cada caso, el contenido de mica, marmolina y cuarzo necesarios para lograr buenas propiedades mecánicas son respectivamente: $5,0 \%, 3,0 \%$ y $7,0 \%$ en peso.

Cada tipo de CMN cambia particularmente la apariencia estética de los materiales compuestos fabricados solo con ATH, permitiendo diferenciarlos visualmente entre sí. En todos los casos, el aspecto visual modificado por las partículas 
proporciona al material compuesto una mejor calidad estética; sin embargo, las propiedades mecánicas disminuyen con la incorporación de dichas cargas respecto al material compuesto fabricado con solo $60 \%$ ATH. Por su apariencia estética, los materiales compuestos con CMN adicionales pueden ser utilizados comercialmente en aplicaciones de menor responsabilidad mecánica.

\section{AGRADECIMIENTOS}

Al Consejo Nacional de Ciencia y Tecnología del Perú (CONCYTEC) y a la Pontifica Universidad Católica del Perú (PUCP) por el financiamiento de la presente investigación (Proyecto de investigación FONDECYT N¹65-2015) y por la beca de maestría otorgada a Kelly Lucas.

\section{BIBLIOGRAFÍA}

[1] ELIAS, H.G., Free Radical Polymerization, In: ELIAS, H.G., Macromolecules: Volume 1: Chemical Structures and Syntheses, 1ed., chapter 20, Weinheim, Germany, Wiley-VCH Verlag GmbH, 2005.

[2] MUNN, G.E., Methacrylate syrups and their preparation, and preparation of reinforced plastic articles employing same, U.S. Patent, n. 3, n. 154, p. 600, 1964.

[3] DUGGINS, R.B., CHADDS, F., Use of alumina trihydrate in a polymer methyl methacrylate article, U.S. Patent, n. 3, pp. 847-865, 1974.

[4] SLOCUM, D.H., Thick, cured polymethyl methacrylate article and process for its preparation, U.S. Patent, n. 3, v. 405, p. $088,1968$.

[5] DUGGINS, R.B., Drive, M., Chadds, F., Process for curing methyl methacrylate in the presence of peroxy catalysts, U.S. Patent n. 3, n. 775, p. 364, 1973.

[6] CHAUDHARY, R., JOSHI, S., SINGH R. C., Mechanical and wear performance of surface composite fabricated by solid-state Technique-A review, Mater. Today Proc., v. 5, n. 14, pp. 28033-28042, 2018.

[7] GUNEL, E. M. BASARAN, C., Micro-deformation mechanisms in thermoformed alumina trihydrate reinforced poly(methyl methacrylate), Mater. Sci. Eng. A, v. 523, n. 1-2, pp. 160-172, 2009.

[8] HULL, T. R., WITKOWSKI, A., AND HOLLINGBERY, L., Fire retardant action of mineral fillers, Polym. Degrad. Stab., v. 96, n. 8, pp. 1462-1469, Aug. 2011.

[9] KUŻDŻAŁ, E., CICHY, B., KICKO-WALCZAK, E., et al.Rheological and fire properties of a composite of unsaturated polyester resin and halogen-free flame retardants, J. Appl. Polym. Sci., v. 134, n. 2, pp. 1-7, 2017.

[10] BERA, P., GUPTHA, N., DASAN, K.P., NATARAJAN, R., Recent developments in synthetic marble processing, Rev. Adv. Mater. Sci., v. 32, n. 2, pp. 94-105, 2012.

[11] ZHANG, R., LI-MAYER, J.Y.S. \& CHARALAMBIDES, M.N. Development of an image-based numerical model for predicting the microstructure-property relationship in alumina trihydrate (ATH) filled poly(methyl methacrylate) (PMMA). Int J Fract, n. 211, pp. 125-148, 2018.

[12] ZHANG, R., Microstructure-property relationships in alumina trihydrate filled poly (methyl methacrylate) composite materials, IOP Conference Series: Materials Science and Engineering, v. 87, 2015.

[13] GUNEL, E., BASARAN, C., Influence of filler content and interphase properties on large deformation micromechanics of particle filled acrylics, Mechanics of Materials, v. 57, pp. 134-146, 2013.

[14] BASARAN, C., NIE, S., HUTCHINS, C. S., \& ERGUN, H.. Influence of Interfacial Bond Strength on Fatigue Life and Thermo-Mechanical Behavior of a Particulate Composite: An Experimental Study, International Journal of Damage Mechanics, v. 17, n.2, 123-147, 2007.

[15] NIE, S., BASARAN, C., HUTCHINS, C. S., \& ERGUN, H., Failure Mechanisms in PMMA/ATH Acrylic Casting Dispersion, Journal of the Mechanical Behavior of Materials, v. 17, n.2, 2006.

[16] A. BYRDY, M. KOŁACZKOWSKI, "Environmental Impacts on the Strength Parameters of Mineral-Acrylic (PMMA/ ATH) Facade Panels,” Int. J. Polym. Sci., v. 2015, 2015.

[17] TUŠAR M., TURK M.R. Laboratory and Field Experience with PMMA/ATH Composite in Asphalt Mixtures. In: Poulikakos L., Cannone Falchetto A., Wistuba M., Hofko B., Porot L., Di Benedetto H. (eds) RILEM 252-CMB Symposium. RILEM 252-CMB 2018. RILEM Bookseries, v. 20, 2019. 
[18] ŠUŠTERŠIČ, E., TUŠAR, M., \& ZUPANČIČ VALANT, A., Rheological and mechanical characterization of waste PMMA/ATH modified bitumen, Construction and Building Materials, v. 38, pp.119-125, 2013.

[19] VOVK, M., PLANINŠEK, O., GERMAN ILIĆ, I., ŠERNEK, M., Characterization of industrial aluminum trihydrate-filled poly(methyl methacrylate) composite powder, Journal of Adhesion Science and Technology, v. 33, n. 23, pp. 25172534, 2019.

[20] ALZARRUG, F.A., DIMITRIJEVIĆ, M.M., JANČIĆ HEINEMANN, R.M., et al., The use of different alumina fillers for improvement of the mechanical properties of hybrid PMMA composites, Mater. Des., v. 86, pp. 575-581, 2015.

[21] LIU, H., YE, H., LIN, ZHOU, T., Synthesis and characterization of PMMA/A12O ${ }_{3}$ composite particles by in situ emulsion polymerization, Particuology, v. 6, n. 3, pp. 207-213, 2008.

[22] ASH, B.J., ROGERS, D.F., WIEGAND, C.J., et al., Mechanical properties of $\mathrm{Al}_{2} \mathrm{O}_{3} /$ polymethylmethacrylate nanocomposites, Polym. Compos., v. 23, n. 6, pp. 1014-1025, 2002.

[23] BILLMEYER, F.W., WILEY J., Textbook of polymer science, 3 ed., New York, U.S.A, Wiley Interscience Publishing Inc., 1984.

[24] WAGNER, H.L., The Mark-Houwink-Sakurada relation for poly (methyl methacrylate), Journal of Physical and Chemical Reference Data, v. 16, n. 2, pp. 165-173, 1987.

[25] REMP. P. Y MERRILL, E., Polymer Synthesis, Second Edition, Huethig and Wepf editors, Basel, 1991.

\section{ORCID}

Adan Smith Arribasplata Seguin

Kelly Lucas Lizano

Juan Carlos Rueda Sánchez

Julio Arnaldo Acosta Sullcahuamán https://orcid.org/0000-0002-2978-1926

https://orcid.org/0000-0001-8280-8326

https://orcid.org/0000-0001-8567-321X

https://orcid.org/0000-0002-1518-4282 